\title{
ASTRONOMY FOR THE BLIND AND THE VISUALLY IMPAIRED
}

\author{
John Gialelis ${ }^{1}$, Andreas Papalambrou ${ }^{1}$ and Aristeidis Hlias ${ }^{2}$ \\ ${ }^{l}$ Electrical \& Computer Engineering Department, University of Patras, Patras, Greece \\ ${ }^{2}$ Department of Informatics, University of Patras, Patras, Greece
}

\begin{abstract}
The need for growth and the social impact of assistive technology for the visually impaired and blind people is driven by the increasing ageing of the population and mainly of the blind population. Blind or visually impaired people cannot visually enjoy the magnificent astronomical objects and the universe and have great difficulty in engaging in the field of astronomy, which makes them feel even more socially excluded than they already are due to their condition, thus, enhancing their social introvertness. This work depicts a system which translates astronomical images into audible sounds. It involves recognition of the astronomical objects of interest inside a picture and separation from background sky and stars as well as detection of their shape, color and brightness. The detected images are then translated into audible sounds and vibration that correspond to the visual information detected. Our approach utilizes principles of state-of-the-art work, thus making sure the proposed system is embraced by the Visually Impaired and Blind People offering them the chance to significantly mitigate their social introvertness.
\end{abstract}

\section{KEYWORDS}

Visually Impaired People, Aural Interpretation of Astronomical Images, Digital Media

\section{INTRODUCTION}

Astronomy can promote and increase public awareness, understanding, and appreciation of science and technology, among people of all ages. Astronomy is an enjoyable, inexpensive hobby for millions of people with great impact on their social inclusion. Blind or visually impaired people cannot visually enjoy the magnificent astronomical objects and the universe and have great difficulty in engaging in the field of astronomy, which makes them feel even more socially excluded than they already are due to their condition, thus, enhancing their social introvertness. Therefore, diminishing this introvertness, by introducing inexpensive and unobtrusive means to blind and visually impaired people to access astronomy is imperative even though viewing an image of an astronomical object is a challenge for disabled people especially for people having vision problems. State of the art digital media can assist people with special needs towards this direction based on existing knowledge that visually impaired people can "see" through sound. Such voice signals exist in traffic lights, for instance, while audio books provide an opportunity to access written material.

The work described in this paper, adheres to well-grounded theory according to which an image of an astronomical object can be conceptualized through a mixture of sounds and leverages contemporary technology, so visually impaired persons can imagine what is being shown (Wagner, 1995, Levy-Tzedek, 2014), and following an innovative methodology of transforming images and photos into sounds, we introduce a system which transforms images into interactive sound files. More specifically, the proposed system can gradually scan an astronomical image and produce a designated sound when the scanning detects the background sky on the image, while when the scanning detects an astronomical object present on the image (e.g. a planet) then the sound characteristics (pitch, loudness, timbre) gradually change increasing or decreasing relatively to the shape, size and brightness of the object. This altering of the sound characteristics can provide an indirect impression of the object to a blind person. Certainly, frequencies and tones that meet the visually impaired preferences are chosen. Apart from the automated scanning option, which is in a way by default set, we provide the option to the user to scroll over with the mouse (or fingers on a touch enabled 
device) on the image, giving thus the ability to explore themselves each image. Thanks to this system, visually impaired people will be capable to "hear" an image but also interact with it as well as enjoy images together with their family and friends. Moreover, a vibrating function assists in easier identification of the borders of astronomical objects.

The rest of the paper is organized as follows. Section 2 briefly describes the background regarding assistive technology (AT) for the visually impaired (VI) and blind people (BP), Section 3 depicts our approach and decomposes our proposed system in its components while Section 4 reveals a demonstration of the proposed system. Section 5 summarizes the progress made so far along with the future steps to follow.

\section{BACKGROUNG AND RELATED WORK}

The need for growth and the social impact of assistive technology (AT) for the visually impaired (VI) and blind people (BP) is driven by the increasing ageing of the population and mainly of the blind population (Hakobyan et al. 2013). Information Technology (IT) was used to give innovative prospects of AT and recent developments on Mobile Devices provide the field of IT-based mobile AT in order to improve the opportunities in quality of life of the visually impaired (Billi et al. 2010, Bhowmick 2017).

By the emergence of the mobile phones, the situation for the VI and BP became more difficult, because the devices needed both visual reading options and handheld operations that were not ergonomic even for people without VI problems. The situation hasn't improved with the modern devices, taking into consideration the requirements of touch and vision being the main sense for their handling (Guerreiro, 2010). The evolution of mobile devices has either built-in the necessary facilities to assist VI and BP, or have been developed auxiliary methodologies and technologies that utilize the abilities of mobile devices with the lack of vision, such as speech recognition, non-speech auditory feedback, voice alerts, things and gesture recognition combining with voice, touch-based feedback, combinations of different sensory modalities, phone camera, etc. (Hakobyan et al. 2013). With ongoing advances in mobile technologies, it is becoming ever more feasible for the VI and BP to rely on mobile devices in order to communicate and understand their ecosystem (Bujacz, 2008).

The field of Human Computer Interaction (HCI) is developing eyes-free interaction methods that can apply to VI and BP, such as Foogue, which can be used to control a mobile device in two modes: menu mode for browsing and controlling a file system that is presented with spatial sound in front of the user, and listening mode, in which music, phone calls, and auditory notifications can be heard simultaneously and positioned around the head of the user (Dicke, 2010). The Slide Rule is a speech-based tool without visual representation. Users navigate through the screen and scan objects by brushing their fingers down the device surface interacting with on-screen objects. It recognizes a one finger scan for browsing information in a text or object list as the user slides a finger in a top-down direction, a second-finger tap for selecting items, a multi-directional flick for performing additional actions, and a L-select gesture for browsing hierarchical information. Its design methodology was user-centralized and came up of formative interviews with VI users (Kane, 2008).

AudioBrowser enables users to be navigate stored information and system commands combining both speech and audio feedback. Users are guided by speech and audio as they move around the screen that is split in two parts in order to allow the user to distinguish between information and commands. As users' fingers move across the screen, non-speech audio is used to inform them when they cross a boundary. Within a given segment of the screen, speech audio informs the user of the information contained therein (Chen, 2006). With the hierarchical structure that supports, the user is able to access different information, such as web pages, text files, audio files, etc. without the need to have visual view on the screen. This approach stemmed from the way VI and BP are looking for information on a touchscreen (Kulyukin, 2011). Another application for touchscreen mobile devices designed to route spatial paths in conjunction with finger-navigation sounds is the Timbremap. If the user wanders off the correct path, audio feedback is generated in order to set the user back to the path to continue tracing. In other mode, Timbremap informs the user about the number of paths around the edges of the screen, about gaps between path segments, and about the existence of any path intersections. While the user navigates the screen, they can hold one finger and by double taping anywhere on the screen with a second finger can listen to available information ( $\mathrm{Su}, 2010)$. Another innovative approach is the Reconfigured Mobile Android Phone (R-MAP). R-MAP is a stand-alone tool for Android 
phones that is based on audio incentives. R-MAP uses the touchscreen of the device, without additional hardware requirements, and its fundamental advantage is that no internet connection is needed. The applications of R-MAP are associated with everyday activities of VI and BP, because it uses to pronounce labels of each packaging. In order for the blind to understand the orientation of the screen and launch the application, there are two buttons on the main activity screen; one in the top right corner of the screen that launch the application, and another in the bottom left corner that enter captured labels. The buttons are accompanied by audio feedback tones (Shaik, 2010).

Our approach utilizes principles of the state-of-the-art work described above, thus making sure the proposed system is embraced by the VI and BP offering them the chance to significantly mitigate their social introvertness.

\section{SYSTEM DESCRIPTION}

The system for aural interpretation of astronomical images translates astronomical images into audible sounds. It involves recognition of the astronomical objects of interest in the picture and separation from background sky and stars as well as detection of their shape, color and brightness. The detected images are then translated into audible sounds that correspond to the visual information detected. The audible sounds produced incorporate three basic elements of human sounds perception that is pitch (related to frequency), loudness (related to intensity) and timbre (related to the qualitative elements of the sounds). These elements are correlated to basic elements of visual perception such as light intensity, color and contrast. The system is provided as a service in the form of an Android application, which displays a full screen image that the users touch with their fingers and swipe across the screen while the system analyses location and speed of the fingers producing the appropriate sounds.

\subsection{Vision and Hearing}

Vision and hearing are the two richest senses of humans, providing a significant variety of sensory stimuli that can transfer a significant amount of information about surroundings including navigation, identification of people and objects, distance calculation, motion detection and much more. Hearing and vision share many similarities but also exhibit significant differences. Vision is based on reception of electromagnetic waves belonging to the visual window of the spectrum by the eye while hearing is based on the reception of mechanical waves travelling through air and belonging to the range of acoustic frequencies. Both vision and hearing can detect information by analyzing the amplitude, the frequency and the qualitative characteristics of waveforms.

A sound has three basic characteristics that are used from the brain to interpret its content. These are loudness, pitch and timbre. Loudness corresponds to the amplitude of the waveform, it's what is called volume in everyday language. Pitch corresponds to the frequency of the waveform, the closest common representation of it being a musical note. The timbre is the most difficult concept because it is defined in an opposite way: what makes two sounds of same volume and pitch apart is their timbre. It can be said that timbre depends on the qualitative characteristics of sound, details in the waveform that can, for instance, tell different instruments apart.

The goal of visual to aural translation is to transfer as much information as possible from the visual field to the aural including both the descriptive aspect as well as the "feeling" of the image.

\subsection{Questionnaire}

In order to better understand the expectations and preferences of the visually disabled, a questionnaire was prepared. The questionnaire contained questions that explored the associations the visually impaired make in their brain between aural and visual concepts, as well as practical issues regarding the format, time duration and complexity of an astronomical visual to aural translation tool. This questionnaire acted as a guide for the basic principles of the system. 
A total of 132 questionnaires were filled in from visually impaired people in 5 countries (Greece, Latvia, France, Cyprus and Poland). $49 \%$ of the participants had a severe or total visual impairment while $51 \%$ had a low or moderate impairment. $42 \%$ of the participant were born with the impairment while $58 \%$ acquired it later on in life. The basic results of the questionnaires show that:

- The system should provide some kind of enjoyment and not be strictly educational.

- $\quad$ Each astronomical picture could be examined by the user for a period of 5-10 minutes.

- The visually impaired have a good understanding of principles such as size and shape but have difficulty understanding the concepts of color and brightness.

\subsection{Conversion}

The visual to aural conversion takes place by associating aural and visual properties as follows. Brightness is correlated to loudness (sound volume) while color is correlated to the sound frequency (pitch) using the correlations of the Sonochromatic Music Scale (Garcia, 2011, Modern Painters, 2008). Shape is perceived with the aid of vibration. The device vibrates briefly when the user transits from the background to the inside of the shape.

\subsection{System Design}

The proposed system comprises three (3) components as depicted below:

$\mathrm{C} 1$ : User input detection and analysis component

This component detects and analyzes user touch input. Parameters of interest to detect are location, speed, acceleration, pressure, and single/multi touch. The analysis of the user input results in determining the proper visual/aural resolution to be used at any given time, e.g. slow finger movement will result in high aural resolution.

C2: Visual to aural translation component

This component makes a real-time visual to aural translation based on pre-calculated associations that are given in three (or more) different resolutions and stored in a table. As an input, this component takes the results of the $\mathrm{C} 1$ and associates user touch pattern into sound specifications based on pre-defined associations. The predefined analysis results for different visual/aural resolutions for each image are provided off-line and do not form part of this component.

C3: Sound generator component

This component generates the actual sounds based on the results of $\mathrm{C} 2$ in two ways:

-Generating tones/sounds based on software sounds generator.

-Generating sounds based on pre-recorded audio library.

\section{IMPLEMENTATION}

\subsection{Astronomical Object Database and Image Preprocessing}

In order to convert images to sound, the basic properties of the image that will be transformed to sound needed to be extracted in discrete way. These properties are shape, brightness and color. MATLAB software was used to extract these properties using algebraic calculations after converting the images to matrices.

To demonstrate the image preprocessing procedure, a sample image, as shown in Figure 1, is used of a globular cluster. 


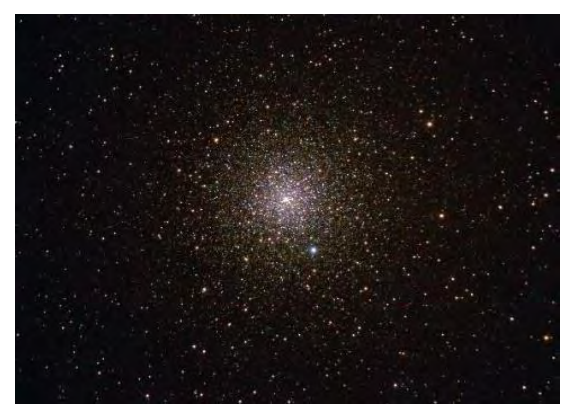

Figure 1. Sample Image

In the first step, a contour tracing algorithm analyzes the image and identifies the contours of the various components of the image as Figure 2 depicts. These can be used for marking the borders of each component to the user. In order to mark the color information of the image, the color resolution is reduced in order to avoid an overload of information in the acoustic field. An algorithm calculates the dominating color of each 20x20 area and replaces all pixels of the area with the dominant color.

Figure 2. Image Contour Identification

A further color simplification, as shown in Figure 3, is performed by reducing the number of colors to the 7 colors of the rainbow.

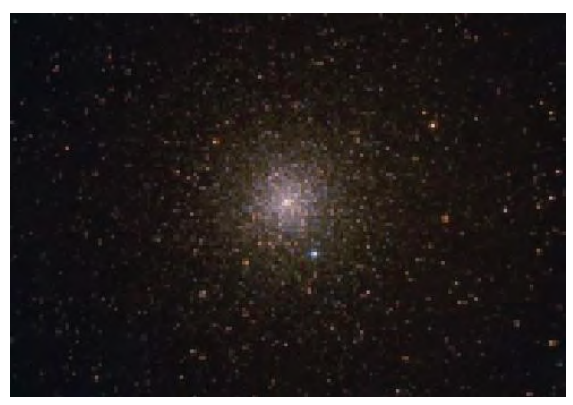

Figure 3. Color Simplification

\subsection{User Interface}

The interface is user friendly and manageable and consists of simple touch commands and a vibration feedback. All screens are voice-assisted for the visually impaired whilst they also depict real images so that they can be used by users with no or non-total visual impairment.

When initially launched, the application presents a voice-guided image selection screen. The user needs only to touch the right image which will be translated to sound, as shown in Figure 4. 


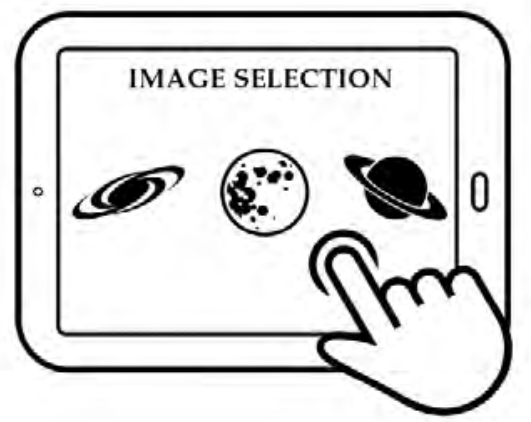

Figure 4. Voice-Guided Image Selection Screen

Once an image is selected, the user can use his finger to navigate the screen. A vibration function aids the user to detect the object edges as shown in Figure 5.

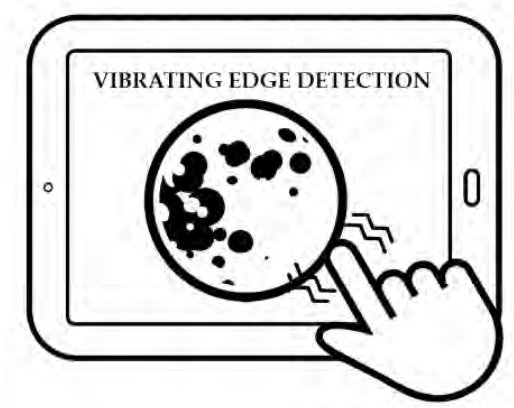

Figure 5. Vibrating Edge Detection Screen

Inside the object borders the app produces sounds as the finger is swiped across the image. The finder swiping speed detection algorithm in combination with the visual to aural translation algorithm, produce sounds that correspond to the visual information at the point of touch as presented in Figure 6.

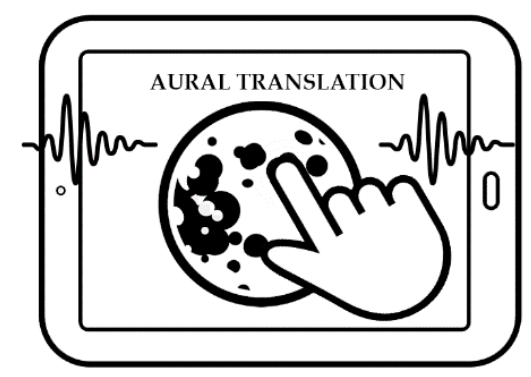

Figure 6. Aural Translation Screen

\section{CONCLUSION}

In this paper the main architectural and implementation aspects of a system, which transforms images into interactive sound files aiming at making visually impaired persons imagine what is being shown, are presented. System's main functions comprise image selection, finger speed detection, edge detection by vibration, finger center point assessment and sound generation. The system has been demonstrated to numerous visually impaired users and feedback has been selected mainly pointing the need for a new sound generation method. More demos are planned to follow so better fine tuning of the application is envisaged. 


\section{ACKNOWLEDGEMENT}

This work is currently supported by the ERASMUS+ Program through the project 2017-1-EL01-KA201-036255-“ASTRONOMY FOR BLIND AND DISABLED (A4BD).

\section{REFERENCES}

Bhowmick, A., \& Hazarika, S. M. (2017), "An insight into assistive technology for the visually impaired and blind people: state-of-the-art and future trends", Journal on Multimodal User Interfaces, Springer, June 2017, Volume 11, Issue 2, pp 149-172.

Billi M, Burzagli L, Catarci T, Burzagli, L., \& Santucci, G. (2010), "A Unified Methodology for the Evaluation of Accessibility and Usability of Mobile Applications", Universal Access in the Information Society, 2010, 9:337-56.

Bujacz, M., Baranski, P., (2008), "Remote mobility and navigation aid for the visually disabled", Proceedings of the 7th International Conference on Disability, Virtual Reality and Assoc. Technologies with Art ArtAbilitation, Maia, Portugal, September 8-11, 2008, 263-70.

Chen, X., Tremaine, M., Lutz, R., Chung, J., Lacsina, P., (2006), "AudioBrowser: a mobile browsable information access for the visually impaired", Universal Access in the Information Society, 2006, 5:4-22.

Dicke, C., Wolf, K., \& Y. Tal (2010), “Foogue: eyes-free interaction for smartphones”, Proceedings of MobileHCI '10, pages 255-248, 2010.

Garcia, F.C "Nace una fundacion dedicada a convertir humanos en ciborgs", La Vanguardia, 1 March 2011.

Guerreiro, T. (2010), "Assessing mobile-wise individual differences in the blind", Proceedings of the 12th International Conference on Human Computer Interaction with Mobile Devices and Services, Lisbon, Portugal, September 7-10, 2010; 485-6.

Hakobyan, L., Lumsden, J., O’Sullivan, D., \& Bartlett, H., (2013) "Mobile assistive technologies for the visually impaired", Survey of Ophthalmology 58, Elsevier, 513-528.

Kane, S.K., Bigham, J.P., Wobbrock, J.O., (2008), "Slide rule: making mobile touch screens accessible to blind people using multi-touch interaction techniques. In Proceedings of the 10th International ACM SIGACCESS Conference on Computers and Accessibility (ASSETS), Halifax, Nova Scotia, Canada, October 13-15, 2008, 73-80.

Kulyukin, V., Crandall, W., Coster, D., (2011), "Efficiency or quality of experience: a laboratory study of three eyes-free touchscreen menu browsing user interfaces for mobile phones", The Open Rehabilitation Journal, 2011, 4:13-22.

Levy-Tzedek, S, Riemer, D, Amedi, A (2014), "Color improves "visual" acuity via sound", Frontiers in Neuroscience, November 2014, Volume 8, Article 358, pp. 1-7.

Modern Painters, The International Contemporary Art Magazine pp 70-73 (New York City, June 2008).

Shaik, A.S., Hossain, G., Yeasin, M. (2010), "Design, development and performance evaluation of reconfigured Mobile Android Phone for people who are blind or visually impaired", Proceedings of the 28th ACM International Conference on Design of Communication (SIGDOC ), Sao Carlos-Sao Paulo, Brazil, September 26-29, 2010, 159-66.

Su, J., Rosenzweig, A., Goel, A., De Laraet, E., \& Truong, K.N., (2010) "Timbremap: enabling the visually-impaired to use maps on touch-enabled devices", Proceedings of the 12th International Conference on Human-Computer Interaction with Mobile Devices and Services (Mobile HCI), Lisbon, Portugal, September 7-10, 2010, 17-26.

Wagner, B. V. (1995), "Improving science instruction for students with disabilities", Proceedings of a working conference on science for persons with disabilities. IA: University of Northern Iowa, pp. 80-83. 